\title{
Small bowel intussusception due to metastatic intestinal carcinosarcoma from a pulmonary primary
}

\author{
Ya-Fen Hsu ${ }^{1,3}$ \\ Chi-Yu Huang' \\ Tong-Jong Chen ${ }^{2}$ \\ Yenn-Hwei Chou' \\ 'Department of General Surgery, \\ ${ }^{2}$ Department of Pathology and \\ Laboratory Medicine, Shin Kong Wu \\ Ho-Su Memorial Hospital, ${ }^{3} \mathrm{Graduate}$ \\ Institute of Medical Sciences, Taipei \\ Medical University, Taipei, Taiwan
}

Correspondence: Yenn-Hwei Chou Department of General Surgery, Shin Kong Wu Ho-Su Memorial Hospital, No 95, Wen Chang Road, Shin Lin District, Taipei II I,Taiwan

Tel +88 622833221 I

Fax +88 6228332211

Emailm0010I0@ms.skh.org.tw
This article was published in the following Dove Press journal:

International Medical Case Reports Journal

I5 December 2010

Number of times this article has been viewed

\begin{abstract}
Metastatic small bowel tumors are rarely encountered. They usually present with small bowel obstruction, perforation, bleeding, or, rarely, intestinal intussusception. Only a few case reports have mentioned bowel symptoms due to metastatic malignancies. We report a seldom encountered clinical condition of intestinal intussusception from metastatic lung malignancy. Pathology demonstrated both epithelial and mesenchymal content, and the final diagnosis was carcinosarcoma. This case report indicates that intestinal metastases should be considered in the differential diagnosis for patients with lung malignancy and abdominal symptoms.
\end{abstract}

Keywords: carcinosarcoma, intussusception, metastatic pulmonary tumors

\section{Introduction}

Pulmonary carcinosarcoma is a rare lung malignancy with aggressive characteristics and a poor prognosis. It accounts for only $1.3 \%$ of lung malignancies. Lung carcinosarcoma occurs mainly in elderly men, with a male-to-female ratio of 7.25:1 and a median age at onset of 65 years. The average tumor size at diagnosis is $7 \mathrm{~cm}$. The five-year survival rate is around $21 \%$. It is usually metastasized at diagnosis. There are rare reports of small intestinal intussusception caused by metastatic lung carcinosarcoma. ${ }^{1}$

\section{Case report}

An 84-year-old male was referred to an emergency room with abdominal fullness and coffee ground vomitus. He presented with nausea and vomiting for two days, with passage of tarry stools. He had taken only small amounts of food in the previous week. A review of his medical records revealed that he had undergone subtotal gastrectomy and a Billroth II gastrojejunal anastomosis for gastric cancer 38 years earlier. In addition, he had undergone transurethral retrograde prostatectomy for prostate cancer 30 years earlier.

At presentation, routine blood and chemistry tests showed white blood cells $30,400 / \mathrm{mm}^{3}$ (segment 80.5), hemoglobin $10.8 \mathrm{~g} / \mathrm{dL}$, C-reactive protein $17.3 \mathrm{mg} / \mathrm{dL}$, blood urea nitrogen $24 \mathrm{mg} / \mathrm{dL}$, creatinine $0.8 \mathrm{mg} / \mathrm{dL}$, sodium $137 \mathrm{mmol} / \mathrm{L}$, and potassium $4.6 \mathrm{mmol} / \mathrm{L}$.

Physical examination revealed a palpable dilated bowel loop in the left lower abdomen. Abdominal sonography demonstrated a typical "donut sign" at axial view and "pseudokidney sign" at longitudinal view (Figure 1A). Small bowel intussusception was suspected. Further imaging studies with abdominal computed tomography (CT) showed multiple protruding small bowel tumors with enteroenteric intussusceptions 


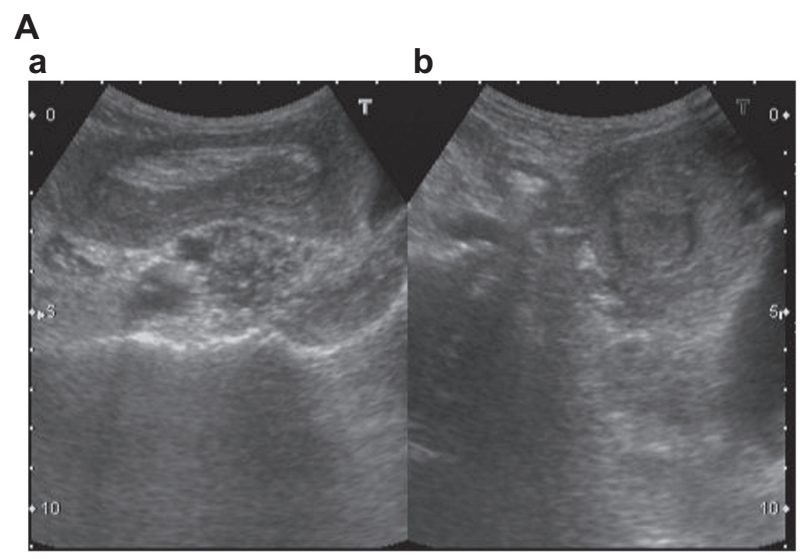

B

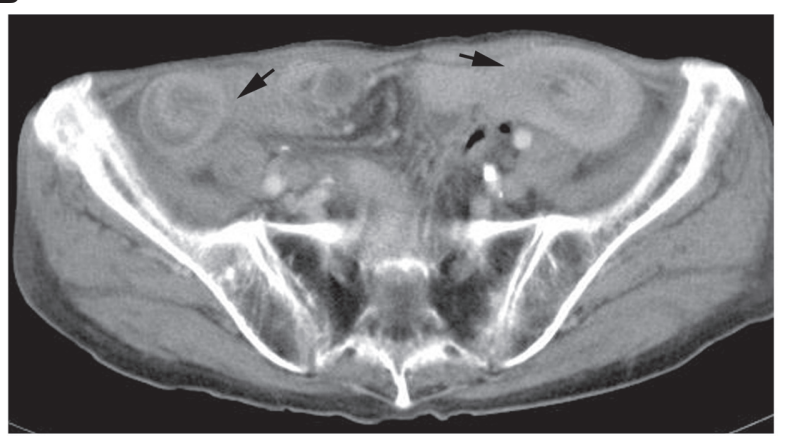

Figure I A) Abdominal ultrasound demonstrating a) "pseudokidney sign" and b) "donut sign". B) Abdominal computed tomography showing bowel intussusception.

at two sites (Figure 1B). Another bulging submucosal tumor was noted incidentally at the gastric fundus. His medical notes showed that he had undergone panendoscopy when coffee ground vomitus had been documented two weeks earlier. This demonstrated a smooth-surfaced bulging tumor without ulceration at the afferent loop below the gastrojejunal anastomosis. Another bulging tumor was seen at the efferent loop $50 \mathrm{~cm}$ distal to the anastomotic site (Figure 2). The tumors had a more intact mucosal surface and looked to have originated from the submucosal layer. A biopsy was taken and the pathology report favored adenocarcinoma (Figure 3). The clinical impression was that of a remnant gastric cancer with intestinal metastases.

Emergency laparotomy performed after the CT scan found two sites of enteroenteric intussusception with proximal small bowel dilatation. The small one was reduced spontaneously by manipulation at laparotomy. The larger one, located in the left lower abdomen, had a sausage-like appearance and resulted in proximal jejunal dilatation (Figure 4A). Meanwhile, there were two tumors with the appearance of serosal invasion, with one located at the afferent loop and the other located at the efferent loop near the gastrojejunal anastomotic site. Careful palpation of the whole length of

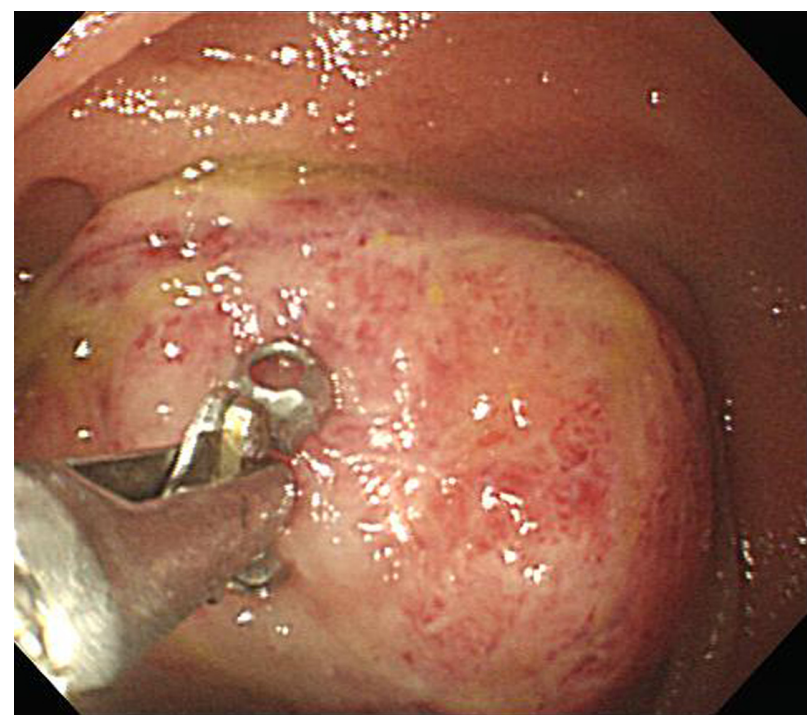

Figure 2 A smooth-surfaced protruding mass without ulceration at the afferent loop just below the gastrojejunal anastomosis. Biopsy favored adenocarcinoma.

the small bowel demonstrated more than three intraluminal tumor-like masses distal to the intussusception site. Revision of gastrojejunostomy with segmental resection of the afferent loop and efferent loop, including the tumor masses, and Roux-en-Y gastrojejunostomy were performed.

The specimen revealed a fungating tumor at the afferent loop and seven cauliflower-like tumors at the efferent loop. Three of the seven tumors resulted in the larger intussusception (Figure 4B). At the same time, a large lymph node up to $1.5 \mathrm{~cm}$ in size at the mesenteric root was excised for pathologic examination.
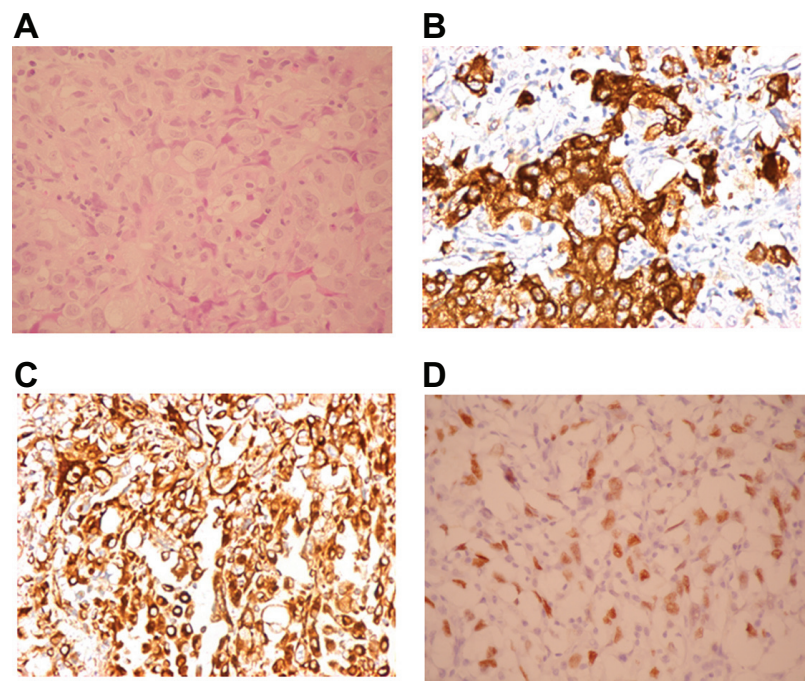

Figure 3 Tumor pathologic characteristics. A biopsy report favored adenocarcinoma $\mathbf{A}$ ). Immunohistological staining showed positivity to the epithelial marker, cytokeratin B) and positivity to the mesenchymal marker, vimentin C). D) A thyroid transcription factor-I-positive stain indirectly confirmed the lung origin. 


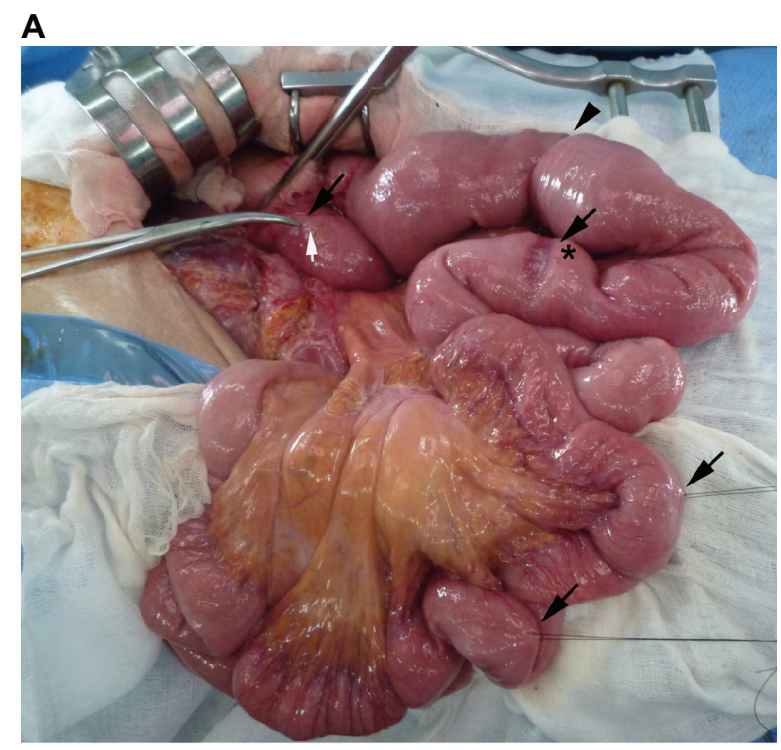

B

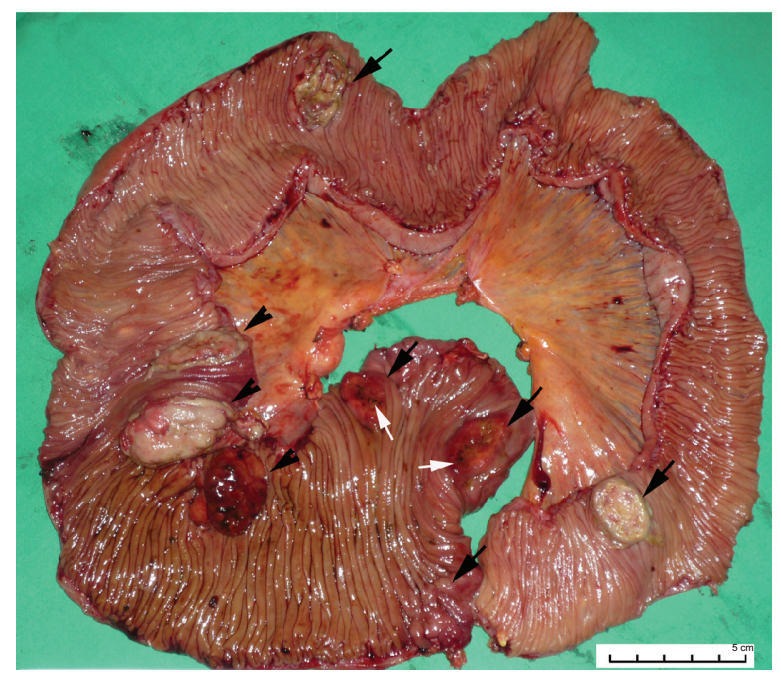

Figure 4 A) Laparotomy demonstrated small bowel intussusceptions (arrowhead) and a reduced second site of intussusception (asterisk). Multiple intraluminal tumors (black arrows) with appearances of serosal invasion (white arrows) and B) multiple fungating tumors (black arrows) with the appearances of gross serosal invasion (white arrows). Three of these contributed to small bowel intussusceptions (arrowhead).

The impression was of metastatic small bowel tumors and mesenteric lymph nodes with an unknown primary. Review of previous medical records and imaging studies showed that a progressively enlarging left upper lung tumor had been found in the preceding six months (Figure 5A and 5B). Pathology showed tumor cell infiltration through the whole thickness of the bowel wall in all eight tumors. They contained both epithelial and mesenchymal elements (Figure 3B and 3C). Special staining demonstrated positivity for thyroid transcription factor-1, indirectly suggesting that the tumors were of lung origin (Figure 3D). The final diagnosis was metastatic small bowel carcinosarcoma with a lung primary.
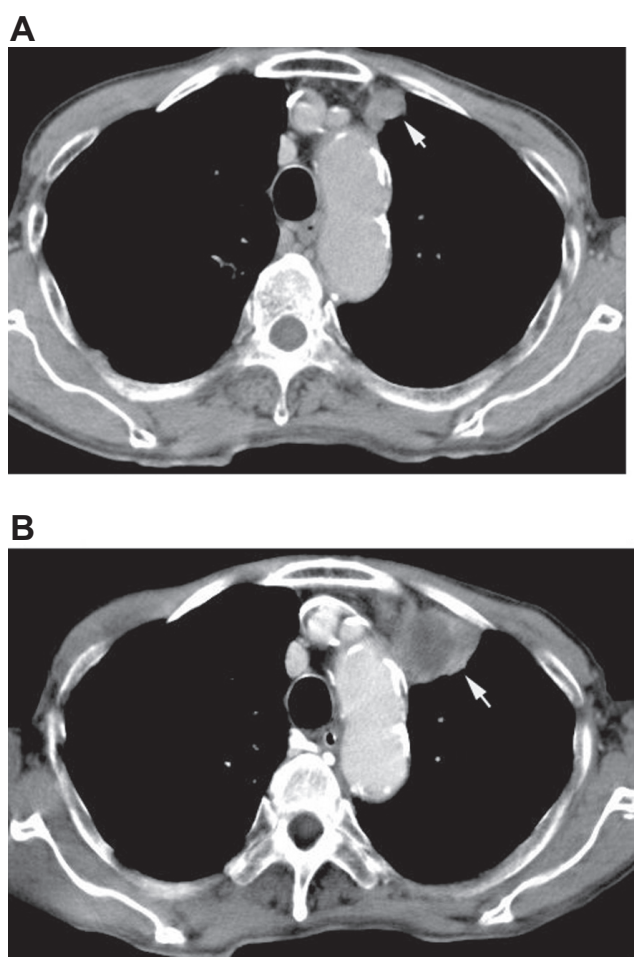

Figure $\mathbf{5}$ Chest computed tomography revealed left upper lung mass (white arrow) half a year previously $\mathbf{A}$ ) and at admission $\mathbf{B}$ ).

Due to respiratory failure after the operation, a lung tumor biopsy was refused by the patient's family. Unfortunately, poor nasogastric feeding and tarry stool passage were noticed 30 days after surgery. Follow-up abdominal CT showed an intra-abdominal abscess, possibly due to anastomotic leakage. Emergency laparotomy demonstrated necrosis at the medial site of the gastrojejunostomy with abscess formation. Primary repair and adequate drainage were performed due to unstable vital signs. The patient died in multiple organ failure one month later.

\section{Discussion}

Ileus is a commonly encountered postoperative complication in the emergency room. The etiologies of small bowel ileus include postoperative tissue adhesion, internal herniation, volvulus, bezoar, and benign or malignant tumors. Adhesion ileus is the leading cause of small bowel obstruction in adults. Although intussusception is one of the leading causes of small bowel obstruction in children, it only accounts for $5 \%$ of small bowel obstruction in adults, and about $90 \%$ of these are secondary. ${ }^{2}$

Patients with ileus experience abdominal distension and fullness for days to months. The presence of nausea and vomiting depends on the level of obstruction in the small bowel. The present case reports a rare condition of small bowel 
obstruction due to enteroenteral intussusception. CT provides the most effective and accurate diagnosis. ${ }^{2}$ The indication for surgery depends on CT findings of bowel obstruction or leading points, ${ }^{3}$ which are mostly benign neoplasms.

Previous papers have demonstrated a few cases of metastatic small bowel cancers from other primaries that include lung origin. The most common clinical presentation of metastatic small bowel tumors is abdominal pain. ${ }^{4}$ Related bowel complications are perforation, enteroenteral intussusceptions, hemorrhage, and obstruction. ${ }^{5}$ Some authors have concluded that small bowel metastases should be kept in mind in patients with lung cancer presenting with abdominal symptoms. Most of these are nonsmall cell lung cancer. ${ }^{4}$

The definitive diagnosis relies on pathologic confirmation. The histology in this case demonstrated a poorly differentiated epithelial component stained with anticytokeratin and a sarcomatous component with antivimentin antibodies. These characteristics made the final diagnosis of carcinosarcoma.

Carcinosarcoma is characterized by both a epitheloid and a mesenchymal component in the same tumor. The sarcomatoid component is thought to be derived from carcinoma through activation of epithelial-to-mesenchymal transition and has the possibility of distant metastases. ${ }^{6}$ Carcinosarcoma has been reported in the uterus and lung, with rare cases in the esophagus, colon, gallbladder, spleen, urinary bladder, and prostate.

Lung carcinosarcoma is a high-grade biphasic neoplasm with both epithelial and mesenchymal components. It usually affects men with a smoking history in the fifth and eighth decades of life and is often refractory to chemoradiation therapy. The survival rate at six months is only around $27 \%{ }^{7}$ This biphasic lung malignancy causes clinical symptoms with chest pain and hemoptysis. The prognosis is worse than for other typical nonsmall cell lung cancers. Because antiepithelial growth factor receptor-targeted therapy provides clinical benefit in patients with nonsmall cell lung cancer, some reports have investigated the potential role of antiepithelial growth factor receptor therapy in patients with lung carcinosarcoma. Most patients are unlikely to benefit from this targeted therapy due to a high rate of K-ras mutation and a rare incidence of increased epidermal growth factor receptor gene copy numbers. ${ }^{8}$

Lung carcinosarcoma with gastrointestinal metastases complicated with intussusception was reported by Eng and Sabanathan in 1992. ${ }^{11}$ Because intestinal metastases usually present in the later stages, they are often accompanied by small bowel obstruction, perforation, ${ }^{9,11}$ bleeding, or, rarely intestinal intussusception. ${ }^{5}$ A previous report demonstrated successful laparoscopic resection of metastatic small bowel carcinosarcoma. ${ }^{10}$

This condition should be differentiated from pulmonary pleomorphic adenoma because there is a different prognosis and outcome. True carcinosarcoma should contain both myoglobin-positive rhabdomyosarcoma and cytokeratinpositive squamous cell carcinoma. Most patients survive for approximately six months after diagnosis.

\section{Conclusion}

Carcinosarcoma is a rare tumor with a poor prognosis. It usually originates from the lung and uterus and is seldom reported in the gastrointestinal tract. The diagnosis is often made at a late stage when there are multiple metastases. However, intussusception is a rare presentation of metastatic pulmonary carcinosarcoma. We should keep in mind bowel intussusception for the differential diagnosis of unusual presentation of ileus. In addition, metastatic small bowel tumors might be reasonably suspected in patients with a history of lung cancer who present with abdominal symptoms.

\section{Disclosure}

The authors report no conflicts of interest in this work.

\section{References}

1. Koss MN, Hochholzer L, Frommelt RA. Carcinosarcomas of the lung: A clinicopathologic study of 66 patients. Am J Surg Pathol. 1999;23:1514-1526.

2. Wang N, Cui XY, Liu Y, et al. Adult intussusception: A retrospective review of 41 cases. World J Gastroenterol. 2009;15:3303-3308.

3. Olasky J, Moazzez A, Barrera K, et al. In the era of routine use of CT scan for acute abdominal pain, should all adults with small bowel intussusception undergo surgery? Am Surg. 2009;75:958-961.

4. Kim SY, Ha HK, Park SW, et al. Gastrointestinal metastasis from primary lung cancer: $\mathrm{CT}$ findings and clinicopathologic features. AJR Am J Roentgenol. 2009;193:W197-W201.

5. Shi B, Gaebelein G, Hildebrandt B, Weichert W, Glanemann M. Adult jejunojejunal intussusception caused by metastasized pleomorphic carcinoma of the lung: Report of a case. Surg Today. 2009;39:984-989.

6. Blaukovitsch M, Halbwedl I, Kothmaier H, Gogg-Kammerer M, Popper HH. Sarcomatoid carcinomas of the lung - are these histogenetically heterogeneous tumors? Virchows Arch. 2006;449:455-461.

7. Vidal-Losada MJ, Bernal-Monterde V, Amores-Arriaga B, et al. Lung carcinosarcoma. Clin Transl Oncol. 2010;12:303-305.

8. Italiano $\mathrm{A}$, Cortot $\mathrm{AB}$, Ilie $\mathrm{M}$, et al. EGFR and KRAS status of primary sarcomatoid carcinomas of the lung: Implications for antiEGFR treatment of a rare lung malignancy. Int J Cancer. 2009;125: 2479-2482.

9. Baldassarre E, Maggi P, Ramieri MT, et al. Jejunal perforation secondary to metastatic sarcomatoid carcinoma of the lung. Minerva Gastroenterol Dietol. 2008;54:225-228. 
10. Felsher J, Brodsky J, Brody F. Laparoscopic small bowel resection of metastatic pulmonary carcinosarcoma. J Laparoendosc Adv Surg Tech A. 2003;13:397-400.
11. Eng J, Sabanathan S. Carcinosarcoma of the lung with gastrointestinal metastasis. Case report. Scand J Thorac Cardiovasc Surg. 1992;26:161-162.

\section{Publish your work in this journal}

The International Medical Case Reports Journal is an international, peer-reviewed open-access journal publishing original case reports from all medical specialties. Previously unpublished medical posters are also accepted relating to any area of clinical or preclinica science. Submissions should not normally exceed 2,000 words or
4 published pages including figures, diagrams and references. The manuscript management system is completely online and includes a very quick and fair peer-review system, which is all easy to use. Visit http://www.dovepress.com/testimonials.php to read real quotes from published authors.

Submit your manuscript here: http://www.dovepress.com/international-medical-case-reports-journal-journal 